\title{
Classification of Benign and Malignant Features of Glioma and Prediction of Early Metastasis and Recurrence Based on Enhanced MRI Imaging
}

\author{
Daiwen Chen (D), Ziqian Chen (D), Shanwen Xu $\mathbb{D}$, and Hui Li $\mathbb{D}$ \\ Department of Diagnostic Radiology, 900 Hospital of the Joint Logistics Team, Fuzhou 350000, Fujian, China \\ Correspondence should be addressed to Daiwen Chen; 1923131207@siit.edu.cn
}

Received 19 December 2021; Revised 9 February 2022; Accepted 12 February 2022; Published 4 March 2022

Academic Editor: M Pallikonda Rajasekaran

Copyright ( 2022 Daiwen Chen et al. This is an open access article distributed under the Creative Commons Attribution License, which permits unrestricted use, distribution, and reproduction in any medium, provided the original work is properly cited.

\begin{abstract}
This work was aimed to establish a feature model for glioma grading and early metastasis and recurrence risk prediction based on contrast-enhanced magnetic resonance imaging (MRI). A total of 145 patients diagnosed with glioma by pathological examination were selected as the research subjects (training cohort: nasty 80; validation cohort: nasty 65). The imaging parameters T1-weighted (CET1WI), axial T2-weighted (T2WI), and apparent diffusion coefficient (ADC) were selected for the extraction of size and shape, intensity, histogram, and texture features. Image dimensionality reduction, feature selection, and model building were performed using the LASSO regression method. Using imaging features as potential predictors and evaluation indicators, the accuracy, sensitivity, and specificity of all prediction models and the area under the curve (AUC) of the receiver operating characteristic curve were calculated. Moreover, a predictive model for glioma grading and early metastasis risk was constructed. The results showed that under a single imaging parameter (T1-CE, DDC, T2WI-FLAIR, ADCslow, Alpha, ADC, CBF, and ADCfast), the diagnostic accuracy, sensitivity, specificity, AUC, and 95\% confidence interval (CI) of low-grade gliomas (LGG), high-grade gliomas (HGG), and recurrent and nonrecurrent gliomas were significantly different $(P<0.05)$. The texture features, histogram features, and mean AUC of distinguishing low-grade and high-grade gliomas were 0.958, 0.945, and 0.954, respectively. The texture features, histogram features, and mean AUC for distinguishing recurrent and nonrecurrent gliomas were 0.949, 0.876, and 0.900 , respectively. In short, the use of enhanced MRI imaging features can realize the prediction of early grading and recurrence of glioma and is of great significance for the early classification of benign and malignant characteristics of tumors.
\end{abstract}

\section{Introduction}

Glioma is the most common primary brain tumor in brain tumors [1]. According to the disease guidance program of the World Health Organization (WHO), gliomas are divided into 4 grades (I-II, low level; III-IV level, and high grade) [2]. The accurate grading of gliomas has important clinical significance for the selection of treatment regimens, monitoring of the efficacy of radiotherapy and chemotherapy, postoperative management of patients, and evaluation of prognosis [3]. In order to better individualize the treatment of patients, it is necessary to accurately predict the prognosis of the disease. Magnetic resonance imaging (MRI) and apparent diffusion coefficient (ADC) can provide important reference criteria for grading the diagnosis of gliomas $[4,5]$.
However, due to the overlap of many similar imaging features of low-grade (LGG) and high-grade (HGG) gliomas on conventional MRI, glioma grading is easily misdiagnosed [6].

The image group is a new field that can convert the texture features of image data into feature values that are easy to synthesize and quantify [7-10] through machine learning algorithms. The imaging group extracts many image texture features from medical images and quantifies the tumor comprehensively through the feature values. Compared with conventional MRI, this technique is a quantitative method and can quantify the phenotypic characteristics of tumors in more detail. The results of radiomics are less disturbed by human factors. At present, radiology is often used to evaluate the clinical phenotype and 
prognosis of lung cancer $[11,12]$. For example, the quantitative features of imaging include many important prognostic indicators, including molecular subtypes of tumors [13]. Although the imaging group has great clinical application value, the imaging technology still has many shortcomings in routine clinical practice. First, the late image processing technology is complex and requires special programs and software. Second, there is no unified standard for the processing of features. Finally, ROI's tumor sketch requires radiologists to draw layer by layer on the image, which is too complex and tedious for clinicians, and there are still errors in the tumor area sketched by each doctor, which leads to differences in feature extraction.

There are studies that use single sequence imaging and single features to grade gliomas to a certain extent [14-16]. However, a single sequence with a single feature cannot quantify all the features of gliomas [17]. However, tumor heterogeneity is an important feature of glioma, and only one MRI sequence cannot extract all the features of glioma. For example, due to various pathological changes in gliomas, such as intratumoral hemorrhage, necrosis, cystic degeneration, and so on, different characteristic signals will be shown by using multisequence MR images. Therefore, the imaging features of multisequence enhanced MR images are helpful in the grading of gliomas as a noninvasive biomarker for preoperative diagnosis of malignant tumors.

Studies suggest that the use of sequences or combinations of multiple imaging features can provide more comprehensive information for glioma grading [18]. Therefore, this work aimed to improve the predictive value of imaging features for early metastasis and recurrence of glioma. In this study, imaging features of multiple contrast-enhanced MRI sequences (such as T2-FLAIR, T1WI-CE, and ADC MAP) were selected for the evaluation of glioma grade and prognosis. The optimal imaging features for each sequence parameter were first determined, and then these features were combined to achieve the best prediction of glioma grade and recurrence. This study aimed to provide a reference for improving the clinical diagnosis, treatment decision, and prognosis prediction value of the disease by using the combination of imaging features.

\section{Materials and Methods}

2.1. Patients and Grouping. In this study, 80 patients with glioma confirmed by pathology after operation in hospital from January 2016 to November 2020 into the training group of this study were included, and 65 patients who met the same inclusion and exclusion criteria in the same period as the verification group. A total of 145 patients were included in this study, including 86 males and 59 females. According to the pathological test results, the patients were divided into high-grade and low-grade groups. Of which, the average age of the high-grade glioma group was $52.4 \pm 15.23$ years old, and that of the low-grade glioma group was $41.0 \pm 13.2$ years old. The magnetic resonance images of glioma patients were collected who meet the above inclusion and exclusion criteria and saved by the picture archiving and communication system (PACS) of the hospital. The study had been approved by the ethics committee of the hospital. The patients and their families had a full understanding of the content and methods of the research and had signed the relevant informed consents.

Inclusion criteria: (1) glioma must be confirmed by postoperative pathology. (2). The images of the subjects were of good quality and had no obvious artifacts. Three conventional MRI sequences (CET1WI, T2WI, and ADC) were available, and all images were obtained within 5 days before surgical treatment.

Exclusion criteria: (1). the patient who was treated before the operation (radiotherapy, chemotherapy, combined radiotherapy and chemotherapy, etc.); (2). without 1.5TMRI examination, the live image quality is poor and there are many artifacts, so it is impossible to carry out this study. All the recurrent cases were confirmed by surgical reexamination, and all recurrent cases were reoperated within one year. The specific recruitment process is as follows (Figure 1).

2.2. Image Data Acquisition. The patients with gliomas were scanned with enhanced MR with an 8-channel phased-array head coil on a 3.0 T high-field magnetic resonance scanner. Continuous acquisition of conventional MR structure images, MR diffusion-weighted images, perfusion-weighted images, and finally the T1 structure images after injection of gadolinium contrast agent. Conventional MR images use fast spin echo sequences (fast spin echo, FSE). (1) axis T1WI: repetition time: $1750 \mathrm{~ms}$, echo time: $25 \mathrm{~ms}$, matrix: $512 \times 512$, scan field (field of view, FOV), $24 \times 24 \mathrm{~cm}$, layer thickness: $5 \mathrm{~mm}$, and layer spacing: $1.5 \mathrm{~mm}$. (2) Axial T2WI: repetition time: $5690 \mathrm{~ms}$, echo time: $93 \mathrm{~ms}$, matrix: $512 \times 512$, FOV: $24 \times 24 \mathrm{~cm}$, layer thickness: $5 \mathrm{~mm}$, and layer spacing: $1.5 \mathrm{~mm}$. (3) After intravenous injection of gadolinium contrast agent ( $0.1 \mathrm{mmol} / \mathrm{kg}$ body weight), the T1WI sequence was scanned repeatedly (the specific parameters were the same as above), and the T1-CE sequence was obtained. The multi-b-value DWI sequence adopts a single-shot plane echo sequence (echo planar sequence, EPI) using $18 \mathrm{~b}$-values in the range of $0 \mathrm{~min} 4500 \mathrm{~mm}^{2} / \mathrm{s}(0 \mathrm{~min} 50,100,150,200,300,500,800$, 1000, 1300, 1500, 2000, 2500, 3000,500, 4000, 4500), NEX: $b=0 \sim 200 \quad(\mathrm{NEX}=1), 300,800 \quad(\mathrm{NEX}=2), \quad 1000,1700$ $(\mathrm{NEX}=4), 2000,4500(\mathrm{NEX}=5)$. Repetition time: $2525 \mathrm{~ms}$, echo time: $88.2 \mathrm{~ms}$, matrix: $256 \times 256$, FOV: $24 \times 24 \mathrm{~cm}$, layer thickness: $5 \mathrm{~mm}$, and layer spacing: $1.5 \mathrm{~mm}$. The thickness of the slices in the above sequence was $4 \mathrm{~mm}$, and the distance between slices was 0 . After MRI collection, the original data generated by 3D-ASL and DWI were used to generate dominant data such as cerebral blood flow (CBF), apparent diffusion coefficient (ADC), and ADC coefficient (eADC) parameter map in the workstation of the GE medical system.

2.3. Tumor Segmentation on Image. The MR images of T2WI-FLAIR, T1WI-CE, and ADC diagrams are transferred from a PACS workstation to a personal computer and then converted into a processable DICOM format image using the RadiAnt DICOM Viewer software (obtained by http:// www.radiantviewer.com). Because of the heterogeneity of gliomas, the region of interest (ROI) was segmented 


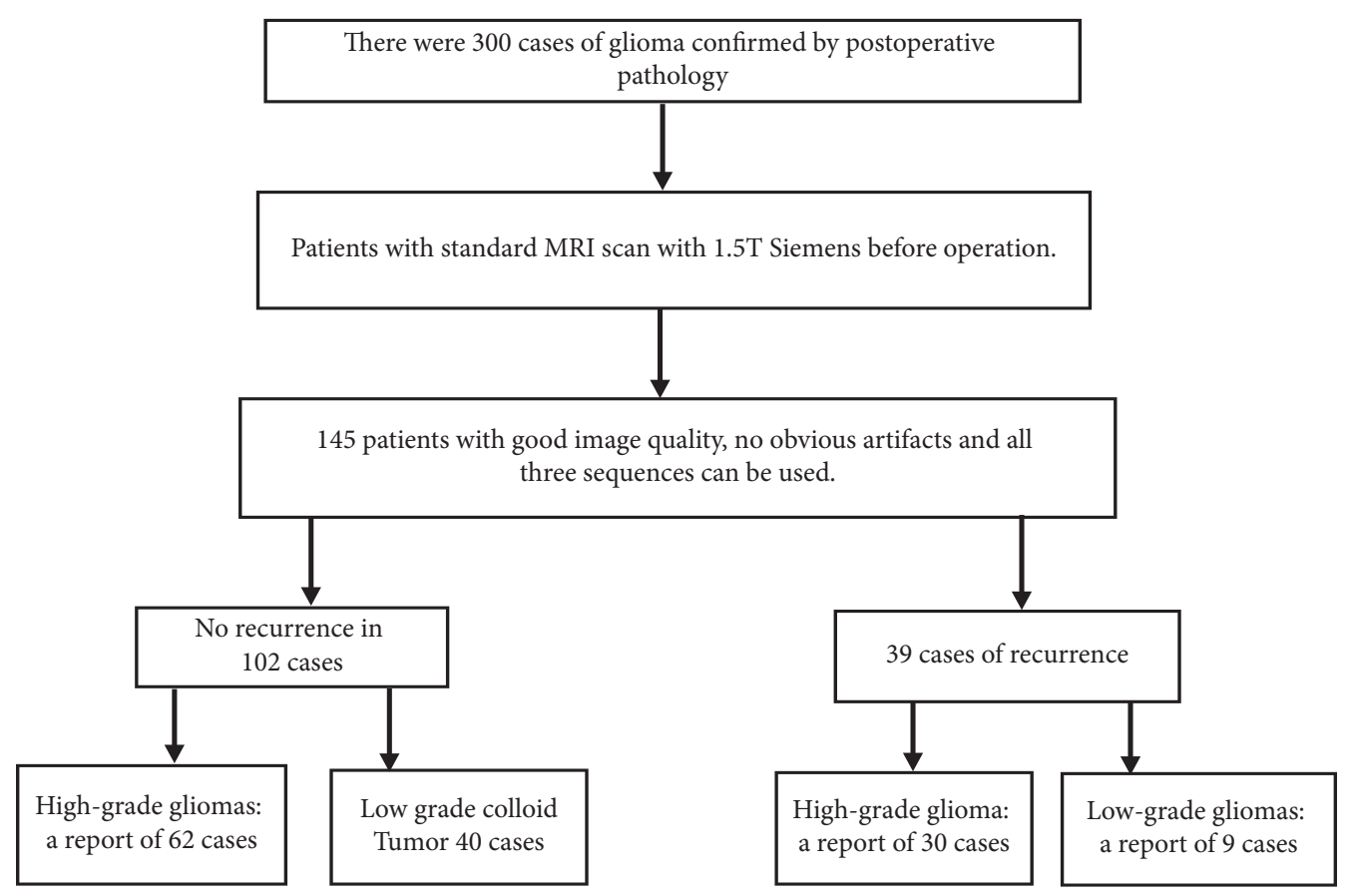

FIgURE 1: Research process.

manually in two-dimensional images using open ITK-SNAP software (http://www.itksnap.org)). Three radiologists with experience in distinguishing neurological tumors by hospital achieved satisfactory manual segmentation until tumors enhanced T1W and T2W FLAIR.

2.4. Image Feature Extraction. All the features are extracted from MATLAB 2016A (MathWorks, Natick, MA) software. The extracted features include Gaussian and Laplace sources (Laplacian of Gaussian, LoG), rotation invariant local binary patterns (rotation invariant local binary patterns, RILBP), gray co-occurrence matrix (GLCM), intensity-based features (IBF), directional wavelet texture feature (directional Gabor texture features, DGTF), and rotation invariant circular wave characteristics (rotation invariant circular Gabor features, RICGF).

\subsection{Radiological Analysis and Processing of Image Data.} It first extracts the radiological features of the enhanced MRI original DICOM image, marks the tumor region on each cross-sectional image of the MRI, and then forms a matrix of all subregions. The matrix sequence is marked with 0 and 1 , respectively, where 0 is nontumor and 1 is a tumor. The matrix generated on the image matrix and the selected region matrix marked $0-1$ is realized by computer program analysis. The program was developed by using the MATLAB script. The relevant radiological features were calculated and extracted from the T2WI-FLAIR, T1WI-CE, and ADC images, respectively. The process of extracting radioactive features is shown in Figure 1. Radioactivity processing produced 152 quantitative image features, which came from the study of three MRI sequences by imaging experts.
2.6. Statistical Processing. SPSS 22.0 was used for the statistical processing of patient information and other data. Continuous variables were expressed as mean \pm standard deviation $(\bar{x} \pm s)$. Differences between two groups were compared using an independent samples $t$ test, and differences between multiple groups were compared using oneway analysis of variance (ANOVA). Dichotomous variables were expressed as frequencies (\%), and differences between groups were compared using the $\chi^{2}$ test. The efficiency of each imaging feature in the diagnosis of early metastasis and recurrence of glioma was analyzed by drawing the receiver operating characteristic curve (ROC) and calculating the accuracy, sensitivity, specificity, and area under the curve (AUC). $P<0.05$ was considered statistically significant.

\section{Results}

First, the differences in baseline data between the LGG group, the HGG group, and the recurrent cases group were compared. Table 1 shows that there was only a significant difference between the average age of the patients in the LGG and HGG groups $(P<0.05)$. Except for the average age, there was no significant difference in other basic data $(P>0.05)$.

145 cases of gliomas were totally resected. The surgical specimens were soaked in $4 \%$ formalin and fixed in paraffin. $1 \mu \mathrm{m}$ sections were prepared and stained with hematoxylineosin (HE). Pathologically, gliomas are classified according to the World Health Organization (2016) central nervous system classification criteria. In addition to the histological grade of the tumor, immunohistochemical (IHC) analysis was also performed. Paraffin sections and routine water dewaxing were performed on the tumor parenchyma, and $\mathrm{ABC}$ immunohistochemical staining was performed. The features were sorted out from the T1-CE, DDC, T2WI- 
Table 1: . Clinical baseline data table of study subjects.

\begin{tabular}{|c|c|c|c|c|c|}
\hline & & LGG & HGG & Recurrent cases & LGG VS HGG $p$ \\
\hline Patients & $(N / \%)$ & $36.55 \%(53 / 145)$ & $64.45 \%(92 / 145)$ & $26.89 \%(39 / 145)$ & NA \\
\hline Age & $\bar{x} \pm s$ & $41.0 \pm 13.2$ & $52.4 \pm 15.23$ & $53.2 \pm 13.78$ & $<0.01^{*}$ \\
\hline Gender & Female $(N / \%)$ & $52.83 \%(28 / 53)$ & $37.0 \%(34 / 92)$ & $35.9 \%(14 / 39)$ & 2.363 \\
\hline \multicolumn{6}{|l|}{ Tumor size $\bar{x} \pm s$} \\
\hline Diameter $(\mathrm{cm})$ & & $4.6 \pm 1.69$ & $5.3 \pm 1.42$ & $5.1 \pm 1.53$ & 2.32 \\
\hline Area $\left(\mathrm{cm}^{2}\right)$ & & $15.2 \pm 9.65$ & $16.56 \pm 10.43$ & $16.21 \pm 9.24$ & 2.56 \\
\hline Volume $\left(\mathrm{cm}^{3}\right)$ & & $36.1 \pm 28.32$ & $44.2 \pm 30.03$ & $38.7 \pm 29.34$ & 1.24 \\
\hline Does the tumor involve gray matter? & & $81.4 \%$ & $73.7 \%$ & $78.3 \%$ & 0.926 \\
\hline Does the tumor cross the midline? & & $18.6 \%$ & $48.2 \%$ & $35.8 \%$ & 2.546 \\
\hline \multicolumn{6}{|l|}{ Tumor location $(N / \%)$} \\
\hline Frontal lobe & & $44.1 \%$ & $50.2 \%$ & $45.7 \%$ & 0.545 \\
\hline Parietal lobe & & $41.7 \%$ & $28.9 \%$ & $33.4 \%$ & 2.67 \\
\hline Temporal lobe & & $5.9 \%$ & $12.2 \%$ & $11.5 \%$ & 1.65 \\
\hline Other location** & & $8.4 \%$ & $8.7 \%$ & $9.4 \%$ & 0.54 \\
\hline
\end{tabular}

Note. In the $p$-value column, $*$ indicated that the difference was statistically significant.

FLAIR, and tumor HE staining of gliomas, and the characteristics were determined by preliminary images of the heterogeneity of tumor imaging, and the postoperative tumor specimens were also attached (Figure 2).

In order to evaluate the superiority of texture features in glioma grading, a series of comparisons were made on the diagnostic effectiveness of texture features, histogram features, and mean features in distinguishing LGG vs. HGG. The texture features, histogram features, and mean features are listed in Figure 3-8, respectively. In the case of every single-mode (T1-CE, DDC, T2WI-FLAIR, ADCslow, Alpha, $\mathrm{ADC}, \mathrm{CBF}$, and ADCfast), the diagnostic accuracy, sensitivity, specificity, AUC, 95\% confidence interval, and corresponding $P$ values of LGG vs. HGG are statistically different. In the case of every single-mode (T1-CE, DDC, T2WI-FLAIR, ADCslow, Alpha, ADC, CBF, ADCfast), the diagnostic accuracy, sensitivity, specificity, AUC, $95 \%$ confidence interval, and the corresponding $P$ value for distinguishing recurrent and nonrecurrent gliomas were statistically different $(P<0.05)$. There was no significant difference except for ADCslow, Alpha, and ADC under histogram characteristics $(P>0.05)$. The areas under the curve of texture feature, histogram feature, and mean feature to distinguish the total features of low-grade and high-grade gliomas were $0.958,0.945$, and 0.954 , respectively. The areas under the curve of texture features, histogram features, and mean features to distinguish early recurrence of gliomas were $0.949,0.876$, and 0.9 , respectively.

\section{Discussion}

Glioma is the most common primary craniocerebral tumor caused by malignant transformation of glial cells in the brain and spinal cord. However, it is necessary to judge whether the glioma is benign or malignant, and the final pathological diagnosis is the gold standard. However because the biopsy is confirmed by biopsy, it is an invasive operation, and the intraoperative sampling can produce negative results [19]. Therefore, there are still limitations in biopsy and diagnosis of benign and malignant tumors. With the brain-enhanced MRI technique in glioma grading and prognosis, the preliminary diagnosis of benign and malignant brain tumors plays a more and more important role in neurosurgical treatment. Recent studies have proved that magnetic resonance imaging is of great value in the metabolic and physiological processes of gliomas, such as DWI [20]. DTI [21], MRS [22, 23], DSC MRI $[24,25]$, and DCE MRI have achieved good results in the evaluation of glioma grading $[26,27]$. In addition, the evaluation of tumor heterogeneity through imaging is of great benefit to guide the clinic, especially in the individualized treatment of gliomas. Therefore, the heterogeneity of gliomas forms a relevant imaging parameter under MRI and can increase the reporting accuracy at the tumor level [28]. This study demonstrates the potential to distinguish between LGG and HGG by enhanced MRI imaging feature extraction without the need for additional imaging examinations. This is a noninvasive and simple step that can be performed on existing DICOM images.

Momeni et al. [29] did a study. According to the ability of the ADC texture feature in MRI images to distinguish HGG from LGG, it is found that the ADC feature can be used to distinguish HGG from LGG, and the feature index of HGG is significantly higher than that of LGG. However, in our study, the combination of multiple features showed a high rate of diagnosis in different grades of gliomas. The diagnosis of the area under the curve under each feature is different, which may be due to the difference between the radiologist's algorithm for feature extraction and the tumor ROI description. In addition, it finds that the entropy of HGG is significantly higher than that of LGG, which is consistent with the previous research results. The texture entropy value of feature extraction is widely used in the index of radiological analysis [30]. Imaging is to extract the relevant imaging features by using the relevant images obtained before the operation, which has been widely used in the diagnosis, treatment, and prognosis of gliomas, and studies have shown that they all have high diagnostic efficiency [31]. In this study, the AUC of the six imaging diagnostic models was all greater than 0.8 , suggesting that the diagnostic efficiency is higher, which verifies the accuracy of the imaging model in the preoperative grading of gliomas. However, all the models have relatively high sensitivity and low 

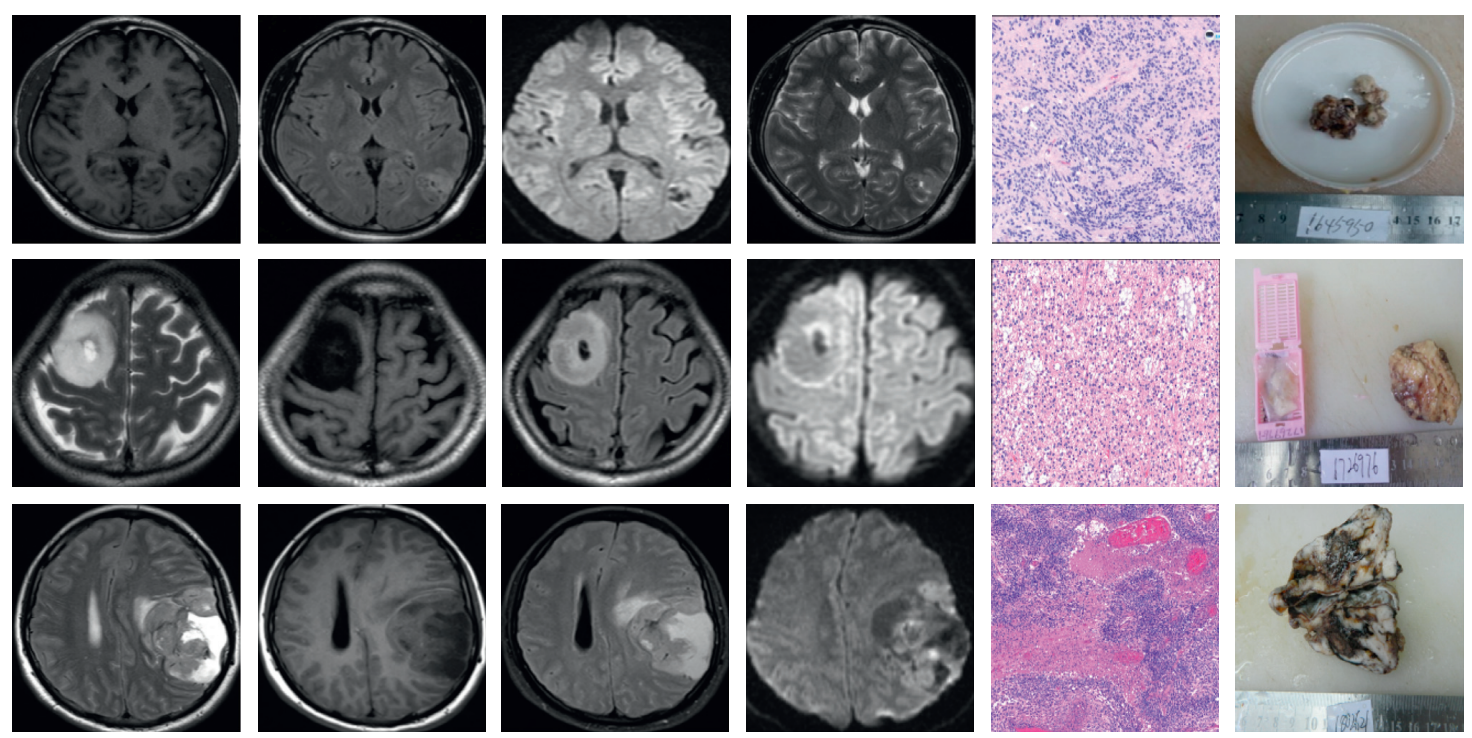

FIgURE 2: Case 1: a 31-year-old male patient underwent resection of the left occipital lobe tumor, and the immunohistochemical results showed angiocentric glioma, WHO grade I; case 2: the frontal lobe tumor of a 55-year-old male patient was resected, and the immunohistochemical results showed ganglion cell glioma (WHO grade II); and case 3: a 16-year-old female patient underwent resection of the tumor in the left temporal lobe, and the immunohistochemical results showed glioblastoma, IDH wild type (WHO grade IV). The order of pictures from left to right was T1WI-CE, T2WI-FLAIR, DDC, T2WI-weight, HE staining, and tumor size measurement.

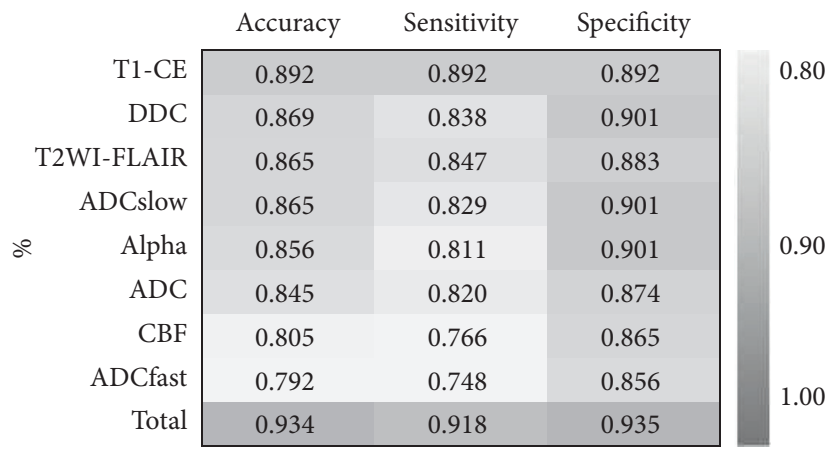

(a)

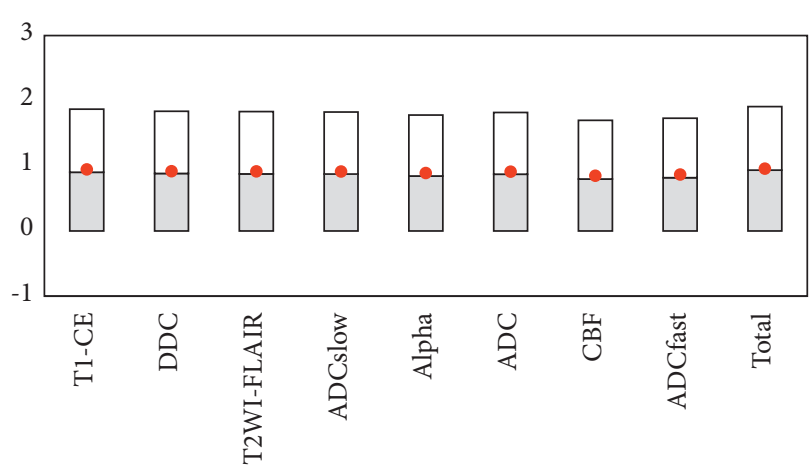

95\% CI_lower limit

口 95\% CI_upper limit

- AUC

(b)

Figure 3: Classification efficiency of texture features for LGG vs. HGG.

specificity, which may be caused by the imbalance of data sample size caused by the difference in the incidence of highand low-grade gliomas. In the future, the model can be trained with a balanced sample size to overcome this difference. At the same time, it observes that on the basis of the same MRI image, the diagnostic efficiency of different machine learning models is different, and the AUC of the RF model based on the T1CE image is the highest, reaching 0.97.

The imaging group is currently a diagnostic method that can quantify the imaging features of different grades of gliomas. Many of the features of these technologies are timeconsuming and difficult to copy. It uses the existing enhanced MRI sequence, which is already a necessary preoperative regimen for gliomas. This is a single-center study that still requires a large sample of clinical data for clinical verification.
At present, biopsy will still be the gold standard, but because brain biopsy is invasive and related to sampling error, this study can be used as a scheme to assist diagnosis, and the diagnostic efficiency can be optimized as part of the imaging contribution of multidisciplinary methods in the diagnosis of gliomas. Further research should focus on optimizing image processing and precise segmentation and also include exploring the characteristics of differences between glioma subgrades because the individualized treatment of glioma patients varies not only between high-grade and low-grade tumors but also between different subgrades. It is believed that the inclusion of nonhistological information, that is, clinical radiology information, can improve clinical diagnosis and biopsy guidance. This means that, in addition to preliminary diagnosis reports, radiologists can also outline the tumor areas 


\begin{tabular}{r|ccc|c}
\multicolumn{1}{c}{} & Accuracy & Sensitivity & \multicolumn{1}{c}{ Specificity } & \multirow{2}{*}{0.80} \\
\cline { 2 - 5 } T1-CE & 0.873 & 0.882 & 0.903 & \\
DDC & 0.879 & 0.869 & 0.876 & \\
T2WI-FLAIR & 0.841 & 0.838 & 0.872 \\
ADCslow & 0.857 & 0.814 & 0.894 \\
Alpha & 0.848 & 0.802 & 0.885 & \multirow{2}{*}{0.90} \\
ADC & 0.829 & 0.817 & 0.882 \\
CBF & 0.833 & 0.801 & 0.879 \\
ADCfast & 0.805 & 0.767 & 0.869 & \multirow{2}{*}{1.00} \\
Total & 0.926 & 0.907 & 0.926 &
\end{tabular}

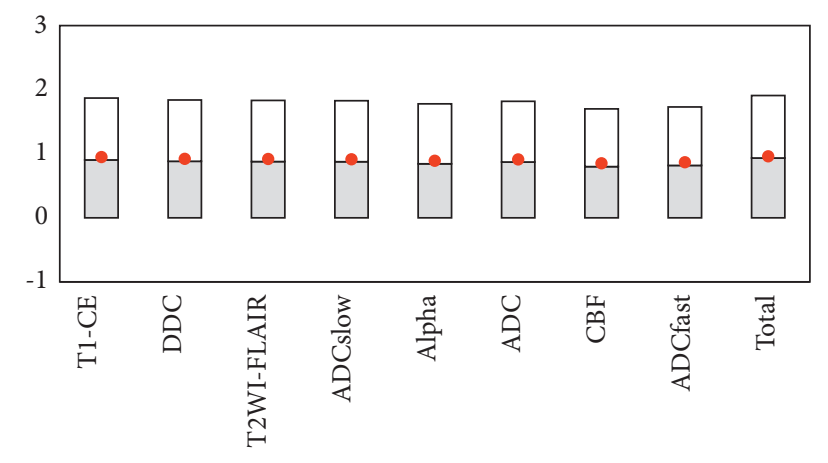
口 95\% CI_lower limit 口 95\% CI_upper limit$$
\text { - AUC }
$$

(b)

FIgURE 4: Predictive efficacy of texture features for recurrence and nonrecurrence of glioma.

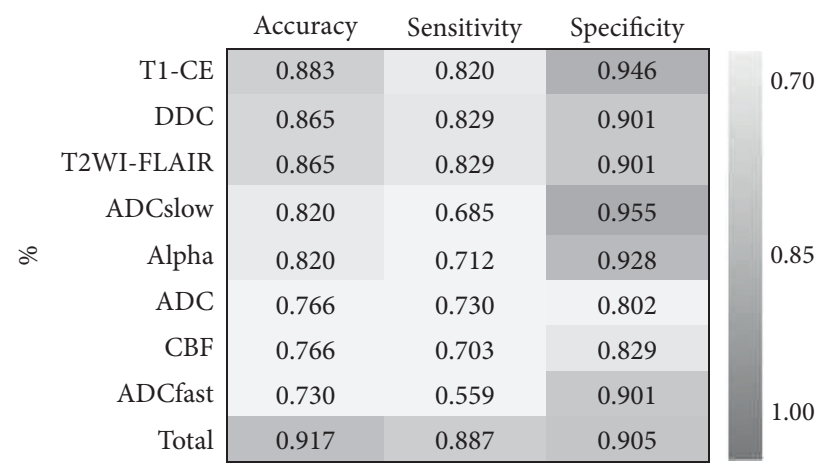

(a)

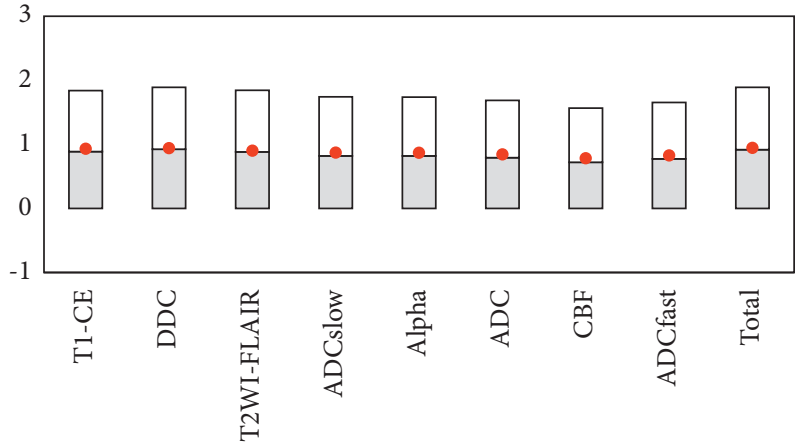

ㅁ 95\% CI_lower limit

95\% CI_upper limit

- AUC

(b)

FIGURE 5: Classification effectiveness of histogram features for LGG vs HGG.

\begin{tabular}{|c|c|c|c|}
\hline & Accuracy & Sensitivity & Specificity \\
\hline T1-CE & 0.698 & 0.631 & 0.766 \\
\hline DDC & 0.667 & 0.500 & 0.838 \\
\hline T2WI-FLAIR & 0.626 & 0.514 & 0.739 \\
\hline ADCslow & 0.613 & 0.712 & 0.514 \\
\hline Alpha & 0.604 & 0.622 & 0.586 \\
\hline $\mathrm{ADC}$ & 0.572 & 0.901 & 0.243 \\
\hline $\mathrm{CBF}$ & 0.698 & 0.631 & 0.766 \\
\hline ADCfast & 0.667 & 0.500 & 0.838 \\
\hline Total & 0.786 & 0.879 & 0.892 \\
\hline
\end{tabular}

(a)

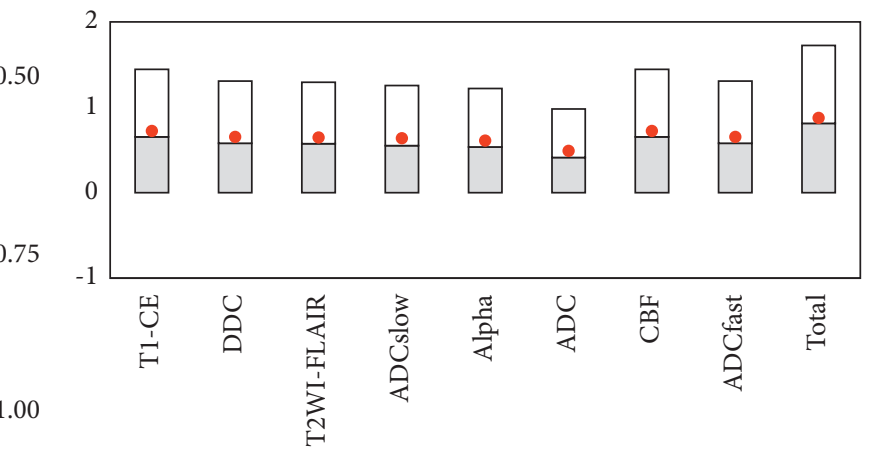

口 95\% CI_lower limit

口 95\% CI_upper limi

- AUC

(b)

FIGURE 6: The effectiveness of texture features in predicting recurrence and nonrecurrence of gliomas. 


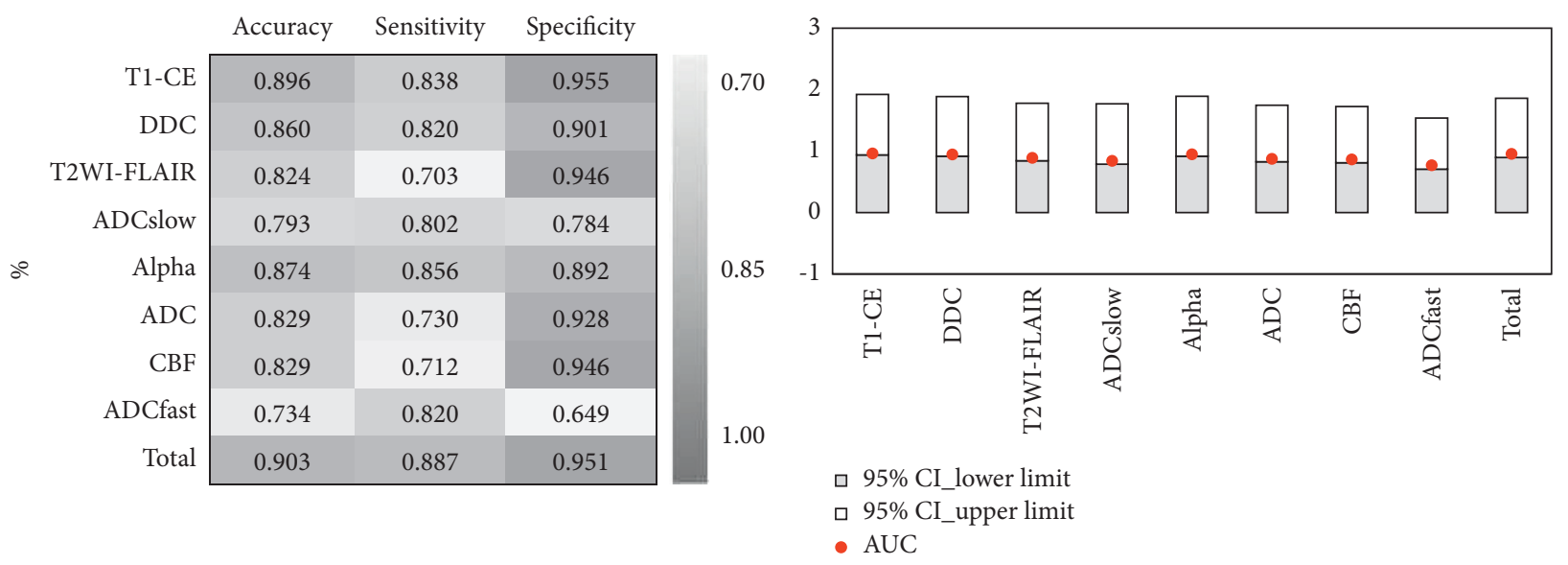

(a)

(b)

Figure 7: Classification efficiency of the mean feature for LGG vs. HGG.

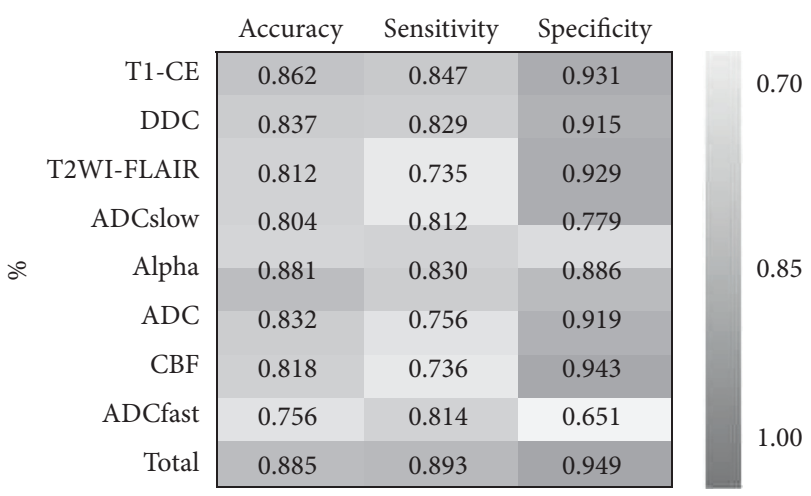

(a)

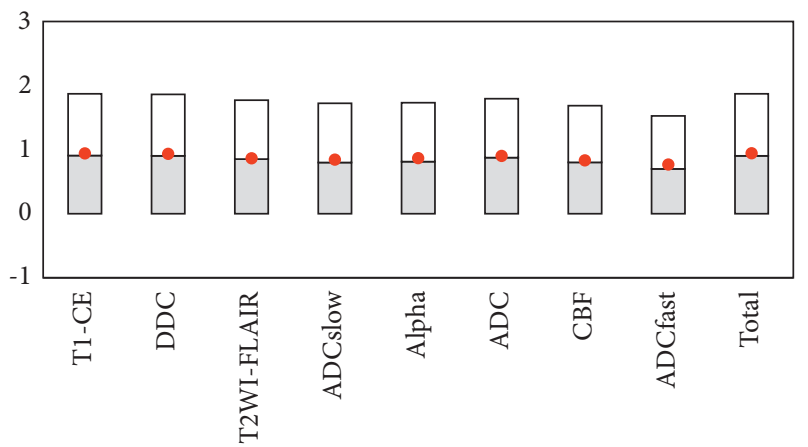

口 95\% CI_lower limit

口 95\% CI_upper limit

- AUC

(b)

Figure 8: Predictive efficiency of mean characteristics for recurrent and nonrecurrent gliomas.

with the highest heterogeneity, helping neurosurgeons to obtain more accurate localization, thus improving the positive rate of samples obtained. At present, MRI feature extraction can be used as a diagnostic grade and prognosis of gliomas, providing a more powerful noninvasive technique and providing a more accurate scientific basis for individual treatment of gliomas [32]. It should be noted that in all of our feature extraction and tumor score ROI, we must strengthen learning so that all feature extraction is more accurate. As we all know, texture feature extraction is often affected by magnetic resonance imaging acquisition, especially the gain factor and spatial resolution [33]. However, there are some limitations to carrying out such research: first, as a pilot study using a picture of a single largest cross section of a tumor, it is difficult to find the precise intraoperative location of its segmented region under a biopsy. This limits the weighting of this study; however, biopsies that obtain accurate localization before tumor resection are usually unlikely. Secondly, the features under MRI can better reflect the heterogeneity of tumors. Even if cases that meet the criteria are selected in the study, HGG is more likely to obtain effective texture features than LGG, which may lead to some wrong results. It tries to reduce the incidence of this situation by using multitexture features. Third, this is a retrospective study, so prospective studies were conducted to verify these results and predict the difference in accuracy between real results and pathology through a large number of clinical samples.

\section{Data Availability}

The data used to support the findings of this study are available from the corresponding author upon request.

\section{Conflicts of Interest}

The authors declare that there are no conflicts of interest.

\section{References}

[1] S. Xu, L. Tang, X. Li, F. Fan, and Z. Liu, "Immunotherapy for glioma: current management and future application," Cancer Letters, vol. 476, pp. 1-12, 2020. 
[2] J. Bai, J. Varghese, and R. Jain, "Adult glioma WHO classification update, genomics, and imaging," Topics in Magnetic Resonance Imaging, vol. 29, no. 2, pp. 71-82, 2020.

[3] R. Ahmed, M. J. Oborski, M. Hwang, F. S. Lieberman, and J. M. Mountz, "Malignant gliomas: current perspectives in diagnosis, treatment, and early response assessment using advanced quantitative imaging methods," Cancer Management and Research, vol. 6, pp. 149-170, 2014.

[4] R. E. Lenkinski, "Spatial resolution versus reproducibility for dynamic MRI of high-grade gliomas," Radiology, vol. 300, no. 2, pp. 421-422, 2021.

[5] P. Korfiatis and B. Erickson, "Deep learning can see the unseeable: predicting molecular markers from MRI of brain gliomas," Clinical Radiology, vol. 74, no. 5, pp. 367-373, 2019.

[6] J. N. Scott, P. M. A. Brasher, R. J. Sevick, N. B. Rewcastle, and P. A. Forsyth, "How often are nonenhancing supratentorial gliomas malignant? A population study," Neurology, vol. 59, no. 6, pp. 947-949, 2002.

[7] P. Lambin, E. Rios-Velazquez, R. Leijenaar et al., "Radiomics: extracting more information from medical images using advanced feature analysis," European Journal of Cancer, vol. 48, no. 4, pp. 441-446, 2012.

[8] H. S. M. Chen, V. A. Kumar, J. M. Johnson et al., "Effect of brain normalization methods on the construction of functional connectomes from resting-state functional MRI in patients with gliomas," Magnetic Resonance in Medicine, vol. 86, no. 1, pp. 487-498, 2021.

[9] P. Lambin, R. G. P. M. van Stiphout, M. H. W. Starmans et al., "Predicting outcomes in radiation oncology-multifactorial decision support systems," Nature Reviews Clinical Oncology, vol. 10, no. 1, pp. 27-40, 2013.

[10] C. Parmar, E. Rios Velazquez, R. Leijenaar et al., "Robust Radiomics feature quantification using semiautomatic volumetric segmentation," PLoS One, vol. 9, no. 7, 2014.

[11] C. Parmar, R. T. H. Leijenaar, P. Grossmann et al., "Radiomic feature clusters and prognostic signatures specific for lung and head \& neck cancer," Scientific Reports, vol. 5, no. 1, Article ID 11044, 2015.

[12] T. P. Coroller, P. Grossmann, Y. Hou et al., "CT-based radiomic signature predicts distant metastasis in lung adenocarcinoma," Radiotherapy \& Oncology, vol. 114, no. 3, pp. 345-350, 2015.

[13] A. Demircioglu, J. Grueneisen, M. Ingenwerth et al., “A rapid volume of interest-based approach of radiomics analysis of breast MRI for tumor decoding and phenotyping of breast cancer," PLoS One, vol. 15, no. 6, 2020.

[14] P. Brynolfsson, D. Nilsson, R. Henriksson et al., "ADC texture-An imaging biomarker for high-grade glioma?” Medical Physics, vol. 41, no. 10, Article ID 101903, 2014.

[15] N. Porz, S. Habegger, R. Meier et al., "Fully automated enhanced tumor compartmentalization: man vs. Machine reloaded," PLoS One, vol. 11, no. 11, 2016.

[16] N. Galldiks, M. Niyazi, A. L. Grosu et al., "Contribution of PET imaging to radiotherapy planning and monitoring in glioma patients - a report of the PET/RANO group," NeuroOncology, vol. 23, no. 6, pp. 881-893, 2021.

[17] M. Law, R. Young, J. Babb et al., "Comparing perfusion metrics obtained from a single compartment versus pharmacokinetic modeling methods using dynamic susceptibility contrast-enhanced perfusion MR imaging with glioma grade," AJNR. American journal of neuroradiology, vol. 27, no. 9, pp. 1975-1982, 2006.

[18] L. L. Wang, J. L. Leach, J. C. Breneman, C. M. McPherson, and M. F. Gaskill-Shipley, "Critical role of imaging in the neurosurgical and radiotherapeutic management of brain tumors," RadioGraphics, vol. 34, no. 3, pp. 702-721, 2014.

[19] M. D. Wood, A. M. Halfpenny, and S. R. Moore, "Applications of molecular neuro-oncology - a review of diffuse glioma integrated diagnosis and emerging molecular entities," Diagnostic Pathology, vol. 14, no. 1, p. 29, 2019.

[20] X. Hu, M. Xue, S. Sun et al., "Combined application of MRS and DWI can effectively predict cell proliferation and assess the grade of glioma: a prospective study," Journal of Clinical Neuroscience, vol. 83, pp. 56-63, 2021.

[21] M. Conti Nibali, M. Rossi, T. Sciortino et al., "Preoperative surgical planning of glioma: limitations and reliability of fMRI and DTI tractography," Journal of Neurosurgical Sciences, vol. 63, no. 2, pp. 127-134, 2019.

[22] Z. Alirezaei, A. Amouheidari, M. Hassanpour et al., "Early detection of radiation-induced injury and prediction of cognitive deficit by MRS metabolites in radiotherapy of lowgrade glioma," BioMed Research International, vol. 2021, Article ID 6616992, 2021.

[23] T. Tong, Z. Yang, J. W. Chen, J. Zhu, and Z. Yao, "Dynamic $1 \mathrm{H}-\mathrm{MRS}$ assessment of brain tumors: a novel approach for differential diagnosis of glioma," Oncotarget, vol. 6, no. 31, Article ID 32257, 2015.

[24] C. H. Sudre, J. Panovska-Griffiths, E. Sanverdi et al., "Machine learning assisted DSC-MRI radiomics as a tool for glioma classification by grade and mutation status," BMC Medical Informatics and Decision Making, vol. 20, no. 1, p. $149,2020$.

[25] J. L. Boxerman, B. M. Ellingson, S. Jeyapalan et al., "Longitudinal DSC-MRI for distinguishing tumor recurrence from pseudoprogression in patients with a high-grade glioma," American Journal of Clinical Oncology, vol. 40, no. 3, pp. 228-234, 2017.

[26] V. C. Keil, G. H. Gielen, B. Pintea et al., "DCE-MRI in glioma, infiltration zone and healthy brain to assess angiogenesis: a biopsy study," Clinical Neuroradiology, vol. 31, no. 4, pp. 1049-1058, 2021.

[27] S. Filice, O. Ortenzia, and G. Crisi, "How tissue T1-variability influences DCE-MRI perfusion parameters estimation of recurrent high-grade glioma after surgery followed by radiochemotherapy," Acta Radiologica, 2021.

[28] F. Davnall, C. S. P. Yip, G. Ljungqvist et al., "Assessment of tumor heterogeneity: an emerging imaging tool for clinical practice?" Insights into Imaging, vol. 3, no. 6, pp. 573-589, 2012.

[29] F. Momeni, R. Abedi-Firouzjah, Z. Farshidfar et al., "Differentiating between low- and high-grade glioma tumors measuring apparent diffusion coefficient values in various regions of the brain," Oman Medical Journal, vol. 36, no. 2, p. 251, 2021.

[30] A. Ditmer, B. Zhang, T. Shujaat et al., "Diagnostic accuracy of MRI texture analysis for grading gliomas," Journal of NeuroOncology, vol. 140, no. 3, pp. 583-589, 2018.

[31] A. S. Jakola, Y.-H. Zhang, A. J. Skjulsvik et al., "Quantitative texture analysis in the prediction of IDH status in low-grade gliomas," Clinical Neurology and Neurosurgery, vol. 164, pp. 114-120, 2018.

[32] D. Aquino, A. Gioppo, G. Finocchiaro, M. G. Bruzzone, and V. Cuccarini, "MRI in glioma immunotherapy: evidence, pitfalls, and perspectives," Journal of immunology research, vol. 2017, Article ID 5813951, 2017.

[33] Q. Tian, L.-F. Yan, X. Zhang et al., "Radiomics strategy for glioma grading using texture features from multiparametric MRI," Journal of Magnetic Resonance Imaging, vol. 48, no. 6, pp. 1518-1528, 2018. 